The authors reported no conflicts of interest.

The Journal policy requires editors and reviewers to disclose conflicts of interest and to decline handling or reviewing manuscripts for which they may have a conflict of interest. The editors and reviewers of this article have no conflicts of interest.

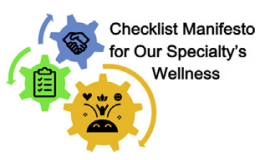

\section{CHECKLIST MANIFESTO FOR OUR SPECIALTY'S WELLNESS}

\section{To the Editor:}

We applaud Fajardo and colleagues ${ }^{1}$ in proposing a wellness policy checklist for cardiothoracic surgery training programs that is sure to be a highly referenced and used resource. ${ }^{2}$ Focusing on the importance of program-level checklists is an important first step. However, we believe there is also a need for checklists and accountability at the individual level to ensure a multifaceted approach to wellness in cardiothoracic surgery. The complementary benefit in fostering both program-level and individual-level checklists in surgery has been demonstrated in the perioperative arena, where preoperative time-outs and checklists have been shown to be effective in improving patient outcomes. ${ }^{3}$

Ensuring wellness at the level of the individual requires taking time to reflect and meditate on our personal goals as often as our professional goals. It entails taking care of our own physical and mental well-being, which ultimately allows us to best serve our patients. We need to cultivate an innate sense of self-awareness to identify what we value, what brings us joy, and to pursue it. When there is room for improvement, seeking help is not a sign of weakness, but a strength, and any stigma toward seeking help must be curbed. Furthermore, there must be recognition that beyond education and equipping one's toolbox with wellness checklists and resources, there is a need for genuine concern for ourselves and others as individuals, and to network, mentor, and sponsor where we can to seek ways to help the next generation.

As a specialty, we need to support trainees and faculty in both their clinical and technical training, but also ensure

\footnotetext{
The Editor welcomes submissions for possible publication in the Letters to the Editor section that consist of commentary on an article published in the Journal or other relevant issues. Authors should: - Include no more than 500 words of text, three authors, and five references. - Type with double-spacing. - See http://jtcs. ctsnetjournals.org/misc/ifora.shtml for detailed submission instructions. • Submit the letter electronically via jtcvs.editorialmanager.com. Letters commenting on an article published in the JTCVS will be considered if they are received within 6 weeks of the time the article was published. Authors of the article being commented on will be given an opportunity of offer a timely response ( 2 weeks) to the letter. Authors of letters will be notified that the letter has been received. Unpublished letters cannot be returned.
}

they find success and wellness in their personal lives. ${ }^{4}$ Our cardiothoracic surgical societies, such as the American Association for Thoracic Surgery, have been active in the development of a Wellness Committee and various scholarship programs to facilitate mentorship to pave the way toward a concerted effort for cultural change whilst cultivating the leaders of tomorrow.

As we seek to build upon the work of Fajardo and colleagues $^{1}$ to construct an individual-level wellness checklist for cardiothoracic surgery, let us ensure that our efforts do not cause evaluation and checklist fatigue, which would defeat the purpose of a wellness policy. In the creation of checklist items, a balance needs to be struck between having sufficient information to be feasible to implement and leaving room to incorporate personal values and goals, while avoiding being so comprehensive that it is overwhelming. If designed thoughtfully, a program-level and individual-level wellness checklist can enhance team cohesion and solidarity through a shared mental model, with resultant effective and sustainable culture change.

The proposed checklist by Fajardo and colleagues ${ }^{1}$ is a great start, and we encourage institutions to use it. Let this be the start of an evolving journey, not the final destination, as we seek and continuously refine our individual and collective approach to optimize our own wellness, and that of our specialty.

Chi Chi Do-Nguyen, $B S^{a}$

Jason J. Han, $M D^{b}$

Jessica G. Y. Luc, $M D^{c}$

${ }^{a}$ Philadelphia College of Osteopathic Medicine

Philadelphia, Pa

${ }^{b}$ Division of Cardiovascular Surgery

Department of Surgery

Hospital of the University of Pennsylvania

Philadelphia, $\mathrm{Pa}$

${ }^{c}$ Division of Cardiovascular Surgery

Department of Surgery

University of British Columbia

Vancouver, British Columbia, Canada

\section{References}

1. Fajardo R, Vaporciyan A, Starnes S, Erkmen CP. Implementation of wellness into a cardiothoracic training program: a checklist for a wellness policy. J Thorac Cardiovasc Surg. 2021;161:1979-86.

2. Han JJ, Kelly JJ, Milewski RK. Burning bright without burning out: protecting the spirit of cardiothoracic surgery. J Thorac Cardiovasc Surg. 2021; 161:339-40.

3. Fajardo R, Vaporciyan A, Starnes S, Erkmen CP. Cardiothoracic surgery wellness: now and the formidable road ahead. J Thorac Cardiovasc Surg. 2021;161:333-7.

4. Cassano AD. Commentary: a wellness checklist for residents is not the only thing that is needed to prevent burnout in the health professional. J Thorac Cardiovasc Surg. 2021;161:1987.

https://doi.org/10.1016/j.jtcvs.2020.07.090 